\title{
Renal Artery Denervation for the Treatment of Hypertension: Opening up New Horizons
}

\author{
Michael Uder • Axel Schmid • Stephanie Titze • \\ Roland E. Schmieder
}

Received: 23 November 2010/Accepted: 9 February 2011/Published online: 1 March 2011

(C) Springer Science+Business Media, LLC and the Cardiovascular and Interventional Radiological Society of Europe (CIRSE) 2011

Hypertension is a major global public health concern. An estimated $30-40 \%$ of the adult population in the developed world suffer from this condition [1,2]. Currently the role of interventional radiology is limited to the few cases in which the renin-angiotensin-aldosterone system is activated by renal artery stenosis. However, stenosis of the renal artery accounts for elevated blood pressure in much $<5 \%$ of patients, and the effect of renal stenting on blood pressure is not as high as expected in the past [3, 4]. In the majority of patients, hypertension is deemed "essential," meaning that no direct cause can be identified and it is believed to be related to both genetic disposition and environmental influences. These patients need lifelong pharmacological therapy. Despite a plethora of antihypertensive drugs, hypertension remains resistant in a considerable number of patients. A new interventional procedure, the catheter-based renal sympathetic denervation (RSD), promises help in such cases of resistant hypertension.

There is increasing evidence that renal efferent sympathetic nerves and afferent sensory nerves that lie within and immediately adjacent to the wall of the renal artery are crucial for initiation and maintenance of systemic hypertension [5-8]. Efferent renal sympathetic activation leads to volume retention via sodium reabsorption, a reduction of renal blood flow by pre- and postglomerular vasoconstriction, and activation of the renin-angiotensin-

\footnotetext{
M. Uder $(\bowtie) \cdot$ A. Schmid

Department of Radiology, University Hospital,

Erlangen, Germany

e-mail: michael.uder@uk-erlangen.de

S. Titze $\cdot$ R. E. Schmieder

Department of Nephrology and Hypertension,

University Hospital, Erlangen, Germany
}

aldosterone system. Afferent renal sensory nerve activity directly influences sympathetic outflow from the central nervous system to the kidneys and other highly innervated organs involved in cardiovascular control, such as the heart and peripheral blood vessels, by modulating hypothalamic activity [9]. Hence, functional denervation of the human kidney by targeting both efferent sympathetic nerves and afferent sensory nerves seems to be a valuable treatment strategy for hypertension [10].

Renal denervation has been used successfully as a therapeutic strategy to prevent hypertension in a variety of experimental models. In humans, radical surgical methods for thoracic, abdominal, and pelvic sympathetic denervation were successfully applied as early as the 1930s to lower blood pressure in patients with malignant hypertension. However, the so-called Smithwick intervention was associated with high perioperative morbidity and mortality and long-term complications, such as bowel, bladder, and erectile dysfunction, and severe postural hypotension [11-13].

For RSD, the treatment catheter (Symplicity, Ardian, Inc., Palo Alto, CA) is introduced into the renal artery via femoral access. Radiofrequency ablations lasting up to 2 min each are applied to four to six discrete points in the renal artery. To destroy the nerve tissue in the whole circumference of the artery, the tip of the ablation wire has to be pulled in a helical manner backward toward the renal artery ostium by $5-\mathrm{mm}$ steps between each ablation. The treatment is analogical to the radiofrequency ablation of aberrant nerve bundles in the heart, which has been performed for many years. At first for safety reasons, the procedure was only performed on one artery per session. After establishing the safety of the technique, a simultaneous bilateral renal artery denervation is normally performed [14]. 
In a first proof-of-principle cohort study, 45 patients were treated with the new method. The baseline mean office blood pressure in the study population was $177 / 101 \mathrm{mmHg}$ (SD 20/15). Office blood pressures after the procedure reduced by $-14 /-10$ (95\% CI 4/3), $-21 /-10(7 / 4),-22 /-11(10 / 5),-24 /-11(9 / 5)$, and $-27 /-17 \mathrm{mmHg}(16 / 11)$ at $1,3,6,9$, and 12 months, respectively [14].

In November, data from a multicenter, prospective, randomized trial were published [15]. In the simplicity HTN-2 trial, 106 patients who had a baseline systolic blood pressure of $160 \mathrm{mmHg}$ or more $(\geq 150 \mathrm{mmHg}$ for patients with type 2 diabetes) were randomly assigned to undergo renal denervation ( $n=52$ ) or not ( $n=54$, control group). The office blood pressure at 6 months in the renal denervation group reduced by $-32 /-12 \mathrm{mmHg}$ (SD 23/11, baseline of $178 / 96 \mathrm{mmHg}, p<0.0001$ ), whereas they did not differ from baseline in the control group (change of $1 / 0 \mathrm{mmHg}$ [21/10], baseline of 178/97 $\mathrm{mmHg}, p>0.05)$. The difference in blood pressure between groups was $33 / 11 \mathrm{mmHg}(p<0.0001)$. At 6 months, $84 \%$ of patients who had undergone renal denervation experienced a reduction in systolic blood pressure of $10 \mathrm{mmHg}$ or more, compared with $35 \%$ of controls $(p<0.0001)$ [15]. The control rate for hypertension, i.e., reduction of systolic blood pressure to $<140 \mathrm{mmHg}$ was $39 \%$ after the renal denervation.

A major question with regard to renal denervation is the durability of the effect on blood pressure. We know from many observations that nerves are occasionally able to regrow anatomically and reinnervate functionally during a period of months to years. An example for complete renal denervation is renal transplantation. However, in humans after transplantation, a recovery of renal sympathetic function has not been shown. In the first pilot study of 153 nonrandomized patients with a follow-up of up to 2 years, no decrease in antihypertensive response was evident after radiofrequency ablation of renal nerves. Eighteen patients of the cohort fulfilled a follow-up of 24 months with a reduction of office blood pressure of $-32 /-14 \mathrm{mmHg}$. In fact, the postprocedure effect on blood pressure increased over time with a reduction of $-20 /-10,-24 /-11$, $-25 /-11,-23 /-11,-26 /-14$, and $-32 /-14 \mathrm{mmHg}$ at 1 , $3,6,12,18$, and 24 months, respectively [16]. The tendency for a progressive decrease in blood pressure over time also was seen in the simplicity HTN-2 trial $(-20 /-7$, $-24 /-8$, and $-32 /-12$ at 1,3 , and 6 months) [15]. It has been speculated that this may represent a predominant alteration in afferent signalling with a resetting of central sympathetic outflow [17].

Despite the amazing clinical success, there are two major concerns with regard to the RSD. One is the safety of the new treatment in terms of procedural and device safety as well as chronic safety. However, the concerns of induction of tissue damage followed by structural changes of renal artery seem to be unfounded. In the HTN-1, one intraprocedural renal artery dissection occurred, but this happened before radiofrequency energy delivery without further sequelae [14]. In HTN-2, no acute renal artery damage was seen [15]. In both the cohort study and in the HTN-2 trial, only a subset of patients was controlled using CT angiography and MR angiography 6 months or more after the intervention. No damage was seen in this subgroup of patients. Hence, mid-term and long-term safety regarding potential damage to the renal artery are still unknown despite no evidence of renal artery stenosis, aneurysm, or other damage have been reported to date [14, 15].

The other concern is the deterioration of renal function due to the procedure itself and associated hemodynamic changes. However, the experience from kidney transplantation in humans, in which sympathetic nerves are completely severed, demonstrates that the denervated kidney is capable of maintaining electrolyte and volume homeostasis. This suggests that selective ablation of renal nerves is unlikely to result in adverse consequences [10]. In the recently published HTN-2 trial, no changes in measured renal function were seen. In particular, in renal-denervation patients with eGFR of $45-60 \mathrm{ml} / \mathrm{min}$ per $\mathrm{m}^{2}$, there was no evidence of worsening function, suggesting that this procedure is safe even in those with mild-to-moderately impaired renal function [15]. The reduction of blood pressure alone would be expected to positively affect renal function. However, the decline in renal function observed in a 24-month follow-up analysis of those treated in the first study (HTN-1) is less than would be predicted based on the blood pressure level achieved [16].

Despite the impressive results of the HTN-2 trial, there are several limitations of the study design. The control group did not undergo sham operation, which would have provided double-blinding and reduced potential bias. It is well known that the prevalence of primary aldosteronism, sleep apnea, and white-coat hypertension is increased in resistant hypertension. Therefore the per-protocol exclusion of secondary and white-coat hypertension would have been more appropriate [18].

The exciting results of the renal denervation therapy generate great expectations regarding the role of interventional radiology for the treatment of systemic hypertension. However, over-optimism has to be dampened. The etiology of hypertension is so complex that the inhibition of one factor can hardly be expected to be effective in all patients. At the moment we only know of unspecific predictors, such as increased baseline blood pressure and reduced heart rate, which do not enable proper patient selection. Considering the limitations of current evidence as of the beginning of 2011, this technique is to be reserved 
to properly assessed, primary hypertensive patients in which medical treatment fails to control BP level. In the future, no one can anticipate the role it will play in other forms of hypertension as well as in other cardiovascular diseases, such as congestive heart failure, chronic kidney disease, diabetes, obesity, and sleep apnea syndrome. There is a need for a huge research effort in this field in the near future and interventional radiology has a significant role to play.

Conflict of interest None.

\section{References}

1. Lloyd-Jones D, Adams R, Carnethon M et al (2009) Heart disease and stroke statistics-2009 update: a report from the American Heart Association Statistics Committee and Stroke Statistics Subcommittee. Circulation 119:480-486

2. Kearney PM, Whelton M, Reynolds K, Muntner P, Whelton PK, He J (2005) Global burden of hypertension: analysis of worldwide data. Lancet 365:217-223

3. Nordmann AJ, Logan AG (2003) Balloon angioplasty versus medical therapy for hypertensive patients with renal artery obstruction. Cochrane Database Syst Rev CD002944

4. Ives NJ, Wheatley K, Stowe RL et al (2003) Continuing uncertainty about the value of percutaneous revascularization in atherosclerotic renovascular disease: a meta-analysis of randomized trials. Nephrol Dial Transplant 18:298-304

5. Schlaich MP, Lambert E, Kaye DM et al (2004) Sympathetic augmentation in hypertension: role of nerve firing, norepinephrine reuptake, and Angiotensin neuromodulation. Hypertension 43:169-175

6. Kopp UC, Cicha MZ, Smith LA, Mulder J, Hokfelt T (2007) Renal sympathetic nerve activity modulates afferent renal nerve activity by PGE2-dependent activation of alpha1- and alpha2adrenoceptors on renal sensory nerve fibers. Am J Physiol Regul Integr Comp Physiol 293:R1561-R1572
7. DiBona GF (2004) The sympathetic nervous system and hypertension: recent developments. Hypertension 43:147-150

8. DiBona GF, Kopp UC (1997) Neural control of renal function. Physiol Rev 77:75-197

9. Doumas M, Faselis C, Papademetriou V (2010) Renal sympathetic denervation and systemic hypertension. Am J Cardiol 105:570-576

10. Schlaich MP, Krum H, Sobotka PA (2010) Renal sympathetic nerve ablation: the new frontier in the treatment of hypertension. Curr Hypertens Rep 12:39-46

11. Evelyn KA, Singh MM, Chapman WP, Perera GA, Thaler H (1960) Effect of thoracolumbar sympathectomy on the clinical course of primary (essential) hypertension. A ten-year study of 100 sympathectomized patients compared with individually matched, symptomatically treated control subjects. Am J Med 28:188-221

12. Morrissey DM, Brookes VS, Cooke WT (1953) Sympathectomy in the treatment of hypertension; review of 122 cases. Lancet 1:403-408

13. Smithwick RH, Thompson JE (1953) Splanchnicectomy for essential hypertension; results in 1,266 cases. JAMA 152:15011504

14. Krum H, Schlaich M, Whitbourn R et al (2009) Catheter-based renal sympathetic denervation for resistant hypertension: a multicentre safety and proof-of-principle cohort study. Lancet 373: 1275-1281

15. Symplicity HTN-2 Investigators (2010) Renal sympathetic denervation in patients with treatment-resistant hypertension (The Symplicity HTN-2 Trial): a randomised controlled trial. Lancet doi:10.1016/S0140-6736(10)62039-9

16. Schlaich M, Krum H, Walton A, Whitbourn R, Sobotka PA, Esler M (2010) Two-year durability of blood pressure reduction with catheter-based renal sympathetic denervation. J Hypertens 28(Suppl A):446

17. Schlaich MP, Sobotka PA, Krum H, Lambert E, Esler MD (2009) Renal sympathetic-nerve ablation for uncontrolled hypertension. N Engl J Med 361:932-934

18. Doumas M, Douma S (2011) Renal sympathetic denervation: the jury is still out. Lancet (in press) 